\title{
Darglitazone Sodium
}

National Cancer Institute

\section{Source}

National Cancer Institute. Darglitazone Sodium. NCI Thesaurus. Code C75188.

The sodium salt form of darg litazone, an agent belonging to the glitazone class of antidiabetic agents with antihyperglycemic and anti-inflammatory activities. Darglitazone may be able to abrogate acute cerebral inflammatory responses via its suppression of proinflammatory gene expression. 